\section{Influence of pupil dilation on retinal nerve fibre layer measurements with spectral domain OCT}

\begin{abstract}
Purpose To evaluate the influence of pupil dilation on retinal nerve fibre layer (RNFL) measurements with spectral domain optical coherence tomography (OCT).

Patients and Methods In total, 29 healthy individuals and 26 glaucoma patients underwent RNFL measurements with the Cirrus HD-OCT Model 4000 (Carl Zeiss Meditec Inc.) before and 30-40 min after pupil dilation with one drop of tropicamide $1 \%$. Average thickness, quadrant thickness, and clock-hour thickness measurements were compared with the paired Student's $t$-test. We also compared the quality scores of the images obtained pre- and post-pupil dilation. Results Mean ages in the glaucoma and control groups were $58.3 \pm 13.4$ and $41.6 \pm 16.4$ years respectively $(P<0.001)$. Mean deviation values were $-6.96+6.31 \mathrm{~dB}$ in the glaucoma group and $-1.26+0.79 \mathrm{~dB}$ in the control group $(P<0.001)$. Mean RNFL measurements obtained in the glaucoma group were significantly lower than those obtained in the control group
\end{abstract} $(P<0.001)$. There were no statistically

Department of Ophthalmology, University of Campinas, Campinas, Brazil

Correspondence: VP Costa, Department of Ophthalmology, University of Campinas, Rua Bahia 1087, apto 21, São Paulo, SP 01244-001, Brazil Tel/Fax: + 551132112000 E-mail:vp.costa@uol.com.br

Received: 7 January 2010 Accepted in revised form: 19 April 2010 Published online: 28 May 2010 significant differences between mean quality scores obtained before and after dilation neither in the glaucoma group $(7.73 \pm 0.92$ vs $7.54 \pm 1.10$, $P=0.232)$ nor in the control group $(8.14 \pm 0.88 \mathrm{vs}$ $8.00 \pm 0.71, P=0.380$ ). There was no statistically significant difference between mean RNFL measurements obtained pre- and post-pupil dilation neither among normal individuals $(P>0.05)$, nor among glaucoma patients $(P>0.05)$.

Conclusion The results of this study indicate that RNFL measurements obtained with spectral domain OCT are not influenced by pupil size. published online 28 May 2010
Eye (2010) 24, 1498-1502; doi:10.1038/eye.2010.72;
GC Massa, VG Vidotti, F Cremasco, APC Lupinacci and VP Costa

Keywords: OCT tomography; mydriasis; glaucoma

\section{Introduction}

Optic nerve and retinal nerve fibre layer (RNFL) imaging have become an important tool in the diagnosis and management of glaucoma. ${ }^{1-6}$ Optical coherence tomography (OCT) is a high-resolution imaging technique that allows in vivo measurements of the RNFL in crosssection without direct contact with the eye. It was introduced in 1991 as a non-invasive technique used initially to investigate macular diseases. ${ }^{5}$ Time-domain OCT, first introduced in $1995,{ }^{6,7}$ has become widely used to obtain RNFL measurements in eyes with glaucoma. ${ }^{8-10}$

Recently, advances in technology allowed the development of spectral-domain OCT (SD-OCT), which provides faster acquisition, better resolution, and improved visualization of retinal morphology. ${ }^{8}$ By using the advanced technology of SD-OCT, the Cirrus HD-OCT acquires data about 70 times faster (27000 vs 400 A-scans per second) and with better resolution (5 vs $\sim 10 \mu \mathrm{m}$ of axial resolution in tissue) when compared to the first generation of OCT. ${ }^{9,11}$ Furthermore, some studies have indicated that time-domain OCT requires pupil dilation for the acquisition of good quality images of the retina and RNFL. ${ }^{11-13}$

Although the manufacturer of the Cirrus HD-OCT indicates that the images can be obtained without pupil dilation, there is no study in the literature investigating the effects of pupil dilation on the measurements obtained with this instrument. Even though there is evidence of the benefits of SD-OCT, factors affecting the variability of measurements should be determined before using it in clinical 
practice. Thus, the purpose of this study was to evaluate the influence of pupil dilation on RNFL measurements obtained with SD-OCT.

\section{Patients and methods}

The study was conducted at the Glaucoma Service of the University of Campinas, Brazil, between April and November 2008, and was approved by the ethics committee of the institution. All participants signed an informed consent before entering the study.

A total of 29 healthy individuals and 26 glaucoma patients were included. All individuals underwent a complete ophthalmological examination including refraction, slit lamp examination, tonometry with the Goldmann applanation tonometer, gonioscopy, indirect ophthalmoscopy, assessment of the optic disc with a $78 \mathrm{D}$ lens, and automated perimetry (SITA or Full threshold strategy, 30-2 or 24-2 program, Humphrey Visual Field Analyzer; Carl Zeiss Meditec Inc., Dublin, CA, USA).

The glaucoma group consisted of patients followed at the Glaucoma Service of University of Campinas. Inclusion criteria for the glaucoma group were: individuals diagnosed with open-angle glaucoma (defined as open angle at gonioscopy, and abnormalities of the optic nerve consistent with glaucomatous damage, with or without visual field loss), corrected visual acuity of 20/40 or better, refractive error $<5$ spherical D and $<3$ cylindrical D, and no history of ocular or systemic disease that could interfere with test results (ie diabetes, temporal arteritis, etc.).

Healthy individuals were selected among friends of patients and the Hospital Staff. The inclusion criteria for healthy volunteers were: no history of eye disease, no family history of glaucoma, intraocular pressure (IOP) $<21 \mathrm{~mm} \mathrm{Hg}$, corrected visual acuity of 20/40 or better, refractive error $<5$ spherical D and $<3$ cylindrical D, open-angles at gonioscopy, normal optic nerve, GHT and PSD within the normal range in automated perimetry.

Both groups underwent RNFL measurements with the Cirrus HD-OCT Model 4000 (Carl Zeiss Meditec Inc.) before and $30-40 \mathrm{~min}$ after pupil dilation with one drop of tropicamide $1 \%$. Subjects were seated with the chin comfortably positioned on a chin rest and the machine properly aligned. The subject was then instructed to look at the internal fixation target to bring the optic nerve head within view of the examiner. The position of the aiming circle was adjusted by the operator to match the optic nerve head best focus and centralization. The repeated scan function was not used. Patients with images with a quality signal lower than 7 were excluded.

The RNFL analyses algorithm reports 17 RNFL thickness values: average RNFL thickness around the entire circumference, average thickness within the four quadrants (temporal, superior, nasal, and inferior), and average thickness in each of the 12 o'clock-hour sectors.

Only one eye per individual was randomly included in the analysis. Left and right eyes were included in the study. Left eyes were considered to be mirror images of the right eyes. Therefore, in all the tables of clock hours, we treated the hours from 1200 to 0600 as nasal, and those from 0600 to 1200 as temporal. Average thickness, quadrant thickness, and clock-hour thickness measurements were compared between the groups. We also compared the quality scores of the images obtained pre- and post-pupil dilation. To investigate the possible influence of the quality score on the variability between pre- and post-dilation measurements, we evaluated the correlation between this variability and the mean quality score of the two measurements.

Categorical variables were compared using the $\chi^{2}$-text or the Fisher's exact test. Continuous variables were compared using the Student's $t$-test or the Mann-Whitney $U$-test. $P$-values of less than 0.05 were considered statistically significant.

We certify that all applicable institutional and governmental regulations concerning the ethical use of human volunteers were followed during this research.

\section{Results}

In total, 29 healthy voluntary individuals and 26 glaucoma patients were included in this study. The demographic characteristics of both groups are shown in Table 1. Mean ages in the glaucoma and control groups were $58.3 \pm 13.4$ and $41.6 \pm 16.4$ years respectively $(P<0.001)$. There was no statistically significant difference regarding gender and race distribution, visual acuity, and refractive error between the groups. The mean IOP was similar in both groups, although the glaucoma group was using a mean number of $2.27 \pm 1.19$ topical medications to lower the IOP.

There was no statistically significant difference between mean quality scores obtained before and after dilation neither in the glaucoma group $(7.73 \pm 0.92$ and $7.54 \pm 1.10, P=0.232)$ nor in the control group $(8.14 \pm 0.88$ and $8.00 \pm 0.71, P=0.380$ ). There was no statistically significant difference between mean RNFL measurements obtained pre- and post-pupil dilation neither among normal individuals $(P>0.05)$, nor among glaucoma patients $(P>0.05$; Table 2$)$. Mean RNFL measurements obtained in the glaucoma group were significantly lower than those obtained in the control group $(P<0.001)$, except for the measurement at the 3 o'clock position (Table 2).

Figure 1 illustrates the relationship between the variability of pre- and post-dilation RBFL measurements and the mean quality score of the two measurements. 
Table 1 Demographics, visual acuity, IOP, and visual field characteristics of both groups

\begin{tabular}{lccc}
\hline & Glaucoma $(\mathrm{n}=26)$ & Healthy individuals $(\mathrm{n}=29)$ & $\mathrm{P}$ \\
\hline Gender (M/F) & $12: 14$ & $12: 17$ & $0.721^{\mathrm{a}}$ \\
Race (C/AA) & $17: 09$ & $25: 04: 00$ & $0.134^{\mathrm{a}}$ \\
Eye (R/L) & $14: 12$ & $19: 10$ & $0.378^{\mathrm{a}}$ \\
Age (years) (mean \pm SD) & $58.3 \pm 13.4$ & $41.6 \pm 16.4$ & $<0.001^{\mathrm{b}}$ \\
Visual acuity -logMAR (mean \pm SD (min; max)) & $0.15 \pm 0.23(0.00 ; 0.90)$ & $0.06 \pm 0.15(0.00 ; 0.70)$ & $0.109^{\mathrm{a}}$ \\
Spherical diopters (mean \pm SD (min; max)) & $0.38 \pm 1.47(-3.75 ; 2.50)$ & $0.49 \pm 1.30(-2.75 ; 4.00)$ & $0.756^{\mathrm{a}}$ \\
Cylindrical diopters (mean \pm SD (min; max)) & $-0.63 \pm 0.67(-2.25 ; 0.00)$ & $-0.46 \pm 0.76(-3.00 ; 0.00)$ & $0.388^{\mathrm{a}}$ \\
Spherical equivalent (mean \pm SD (min; max)) & $0.06 \pm 1.46(-3.75 ; 2.13)$ & $0.26 \pm 1.21(-2.75 ; 3.88)$ & $0.579^{\mathrm{a}}$ \\
IOP (mm Hg) (mean \pm SD (min; max)) & $14.15 \pm 3.53(7.00 ; 21.00)$ & $13.34 \pm 1.93(10.00 ; 18.00)$ & $0.405^{\mathrm{a}}$ \\
MD (dB) (mean \pm SD (min; max)) & $-6.96 \pm 6.31(-22.98 ;-0.46)$ & $-1.26 \pm 0.79(-3.17 ; 0.27)$ & $<0.001^{\mathrm{a}}$ \\
PSD (dB) (mean \pm SD (min; max)) & $5.68 \pm 4.18(0.97 ; 15.17)$ & $1.74 \pm 0.89(1.12 ; 5.60)$ & $<0.001^{\mathrm{a}}$ \\
\hline
\end{tabular}

AA, African American; C, Caucasian; F, Female; IOP, intraocular pressure; L, left; M, Male; MDz, mean deviation; PSD, pattern standard deviation; $\mathrm{R}$, right; SD, standard deviation.

aPearson's $\chi^{2}$-test.

bstudent's $t$-test.

Table 2 Ocular coherence tomography data

\begin{tabular}{|c|c|c|c|c|c|c|c|c|}
\hline & \multicolumn{3}{|c|}{ Glaucoma $(\mathrm{n}=26)$} & \multicolumn{3}{|c|}{ Healthy individuals $(\mathrm{n}=29)$} & \multirow[t]{2}{*}{$\mathrm{P}^{\mathrm{a}, \mathrm{b}}$} & \multirow[t]{2}{*}{$\mathrm{P}^{\mathrm{b}, \mathrm{c}}$} \\
\hline & Undilated (mean $\pm S D)$ & Dilated (mean $\pm S D)$ & $\mathrm{P}^{\mathrm{d}}$ & Undilated $($ mean $\pm S D)$ & Dilated (mean $\pm S D)$ & $\mathrm{P}^{\mathrm{d}}$ & & \\
\hline Q & $7.73 \pm 0.92$ & $7.54 \pm 1.10$ & 0.232 & $8.14 \pm 0.88$ & $8.00 \pm 0.71$ & 0.380 & 0.097 & 0.080 \\
\hline AT & $73.00 \pm 12.58$ & $73.31 \pm 13.33$ & 0.624 & $96.17 \pm 11.59$ & $97.07 \pm 9.16$ & 0.300 & $<0.001$ & $<0.001$ \\
\hline ST & $86.00 \pm 20.08$ & $86.42 \pm 20.82$ & 0.689 & $122.90 \pm 21.17$ & $123.38 \pm 19.31$ & 0.790 & $<0.001$ & $<0.001$ \\
\hline NT & $63.35 \pm 9.09$ & $64.08 \pm 10.19$ & 0.520 & $71.86 \pm 12.15$ & $72.97 \pm 9.88$ & 0.359 & 0.010 & 0.003 \\
\hline IT & $89.19 \pm 20.84$ & $89.23 \pm 20.53$ & 0.963 & $124.59 \pm 19.42$ & $125.34 \pm 14.95$ & 0.723 & $<0.001$ & $<0.001$ \\
\hline TT & $53.42 \pm 12.21$ & $53.46 \pm 13.78$ & 0.971 & $65.38 \pm 9.96$ & $66.45 \pm 11.85$ & 0.256 & 0.001 & 0.001 \\
\hline $\mathrm{H} 1 \mathrm{~T}$ & $80.73 \pm 24.00$ & $79.88 \pm 23.16$ & 0.555 & $113.24 \pm 26.74$ & $116.45 \pm 26.06$ & 0.131 & $<0.001$ & $<0.001$ \\
\hline $\mathrm{H} 2 \mathrm{~T}$ & $76.85 \pm 18.84$ & $75.62 \pm 17.68$ & 0.458 & $86.69 \pm 16.76$ & $90.66 \pm 16.71$ & 0.110 & 0.039 & 0.002 \\
\hline H3T & $55.88 \pm 7.52$ & $57.19 \pm 10.14$ & 0.347 & $60.31 \pm 11.04$ & $60.07 \pm 9.65$ & 0.811 & 0.120 & 0.209 \\
\hline $\mathrm{H} 4 \mathrm{~T}$ & $57.65 \pm 8.46$ & $59.15 \pm 11.01$ & 0.289 & $68.55 \pm 15.77$ & $68.21 \pm 13.14$ & 0.781 & 0.010 & 0.012 \\
\hline H5T & $77.12 \pm 18.69$ & $77.08 \pm 18.97$ & 0.977 & $101.41 \pm 23.32$ & $102.10 \pm 18.96$ & 0.766 & $<0.001$ & $<0.001$ \\
\hline H6T & $95.54 \pm 25.56$ & $95.81 \pm 22.79$ & 0.830 & $137.21 \pm 31.69$ & $136.86 \pm 25.22$ & 0.914 & $<0.001$ & $<0.001$ \\
\hline H7T & $94.65 \pm 31.73$ & $95.00 \pm 32.70$ & 0.816 & $135.07 \pm 19.24$ & $136.97 \pm 24.41$ & 0.386 & $<0.001$ & $<0.001$ \\
\hline H9T & $56.69 \pm 13.23$ & $55.31 \pm 14.97$ & 0.386 & $66.48 \pm 13.63$ & $67.62 \pm 16.15$ & 0.509 & 0.018 & 0.009 \\
\hline H10T & $44.88 \pm 10.94$ & $45.65 \pm 11.80$ & 0.618 & $51.97 \pm 11.74$ & $52.97 \pm 14.71$ & 0.362 & 0.025 & 0.036 \\
\hline $\mathrm{H} 11 \mathrm{~T}$ & $58.69 \pm 19.54$ & $59.46 \pm 21.02$ & 0.401 & $77.45 \pm 12.87$ & $79.10 \pm 14.82$ & 0.230 & $<0.001$ & $<0.001$ \\
\hline $\mathrm{H} 12 \mathrm{~T}$ & $88.46 \pm 32.21$ & $89.35 \pm 33.44$ & 0.499 & $8.14 \pm 0.88$ & $125.76 \pm 22.88$ & 0.846 & $<0.001$ & $<0.001$ \\
\hline $\mathrm{H} 12 \mathrm{~T}$ & $88.77 \pm 22.64$ & $89.96 \pm 21.74$ & 0.452 & $96.17 \pm 11.59$ & $125.41 \pm 23.88$ & 0.701 & $<0.001$ & $<0.001$ \\
\hline
\end{tabular}

$\mathrm{AT}$, average thickness; IT, inferior thickness; NT, nasal thickness; ST, superior thickness; SD-standard deviation; TT, temporal thickness; $\mathrm{Q}$, quality score; H1T to H12T, thickness at 0100, 0200, 0300, 0400, 0500, 0600, 0700, 0800, 0900, 1000, 1100, and 1200 hours sectors.

${ }^{a}$ Comparison between non-dilated glaucoma group $v s$ non-dilated healthy individuals.

${ }^{\mathrm{b}}$ Mann-Whitney $U$-test.

${ }^{\mathrm{c} C}$ Comparison between dilated glaucoma group $v s$ dilated healthy individuals.

'Paired Student's t-test.

The bold numbers represent $P$ values $<0.05$.

There was not a statistically significant correlation between the two parameters neither for the glaucoma group $(r=-0.098, P=0.635)$ nor for the control group $(r=-0.031, P=0.874)$.

\section{Conclusion}

As mentioned above, the development of SD-OCT for RNFL measurements allowed a better resolution and shorter examination time. Numerous reports have compared TD and SD-OCT. ${ }^{14-18}$ Although SD-OCT has shown better reproducibility than TD-OCT, ${ }^{18,19}$ glaucoma discrimination has been found to be similar for TD-OCT and SD-OCT. ${ }^{20}$

Several studies have investigated the influence of pupil dilation on RNFL measurements obtained with TD-OCT. ${ }^{11-13,21,22}$ Smith et al ${ }^{11}$ suggested that the TD-OCT requires pupil dilatation to perform the examination, as the quality of scans and the reproducibility of RNFL measurements is not satisfactory in $25 \%$ of undilated 


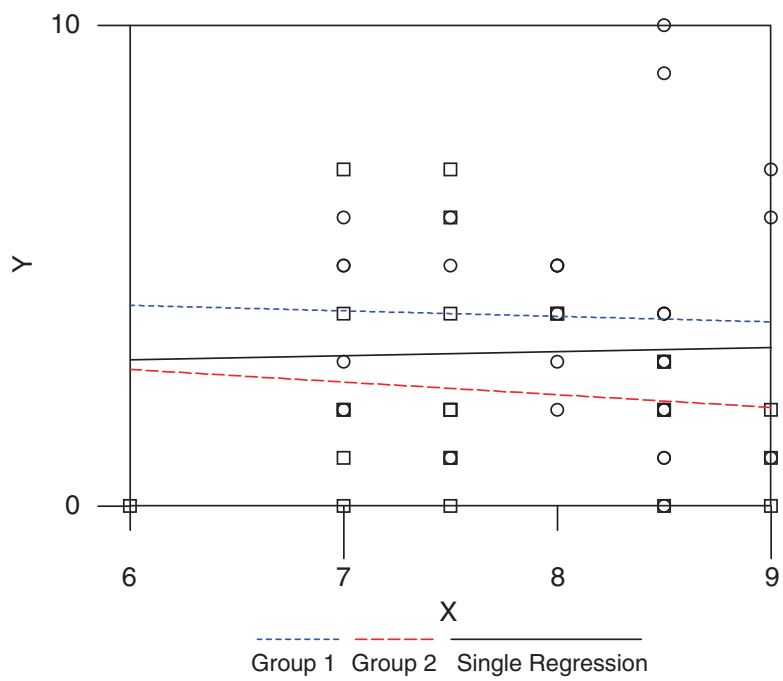

Figure 1 Scatter plot showing the relationship between the preand post-dilation RNFL measurements difference ( $y$ axis) and the mean quality score of the two measurements ( $x$ axis). Group 1, Control group (circles); Group 2, Glaucoma group (squares).

patients. Similarly, Paunescu et $a l^{12}$ have shown that the best reproducibility with the Stratus OCT was obtained under dilated pupils for RNFL, macular, and optic nerve head parameters.

However, Zafar et $a l^{21}$ have shown no significant difference in RNFL thickness measurements obtained with TD-OCT before and after dilation using both fast and regular RNFL protocols. Nevertheless, this study was limited by the use of young and normal subjects rather than glaucoma patients. This young population had relatively large pupils, which may not be present in older individuals or glaucoma patients. According to Hsu and Tsai, ${ }^{22}$ peripapillary RNFL thickness obtained with TD-OCT before and after pupillary dilation in Taiwanese normal individuals showed no significant difference $(P>0.05)$ in average, superior, inferior, temporal, or nasal measurements. Finally, according to Savini et $a l^{13}$ the pupil size only marginally affected RNFL measurements obtained with Stratus OCT.

Even though controversy remains regarding the influence of pupil dilation on the measurements obtained with TD-OCT, there is no study evaluating this possible source of variability with SD-OCT. Our study showed that mean RNFL measurements did not differ before and after pupil dilation either in glaucoma patients or in healthy individuals. According to the manufacturer, Cirrus OCT requires a $2 \mathrm{~mm}$ pupil size, in contrast to the Stratus OCT, which requires a $4 \mathrm{~mm}$ pupil diameter. This difference is due to how the fundus image is created in each instrument. The Cirrus OCT uses an on-axis line camera to scan across the fundus and construct the image from the respective lines taken. The Stratus OCT uses an off-axis CCD camera, and, therefore, needs a wider pupil to allow the rays from the retina to pass.

Our study has one important limitation. We included relatively young individuals with visual acuity better than or equal to 20/40, which certainly eliminated eyes with media opacities or abnormal pupils. Unfortunately, we did not obtain pupil size measurements before and after dilation. Hence, our study results may not be applicable to patients with small pupils or media opacities.

In summary, our findings confirm that pupil dilation is not necessary in most of the eyes undergoing RNFL thickness measurements with SD-OCT. Because pupil dilation is time consuming, induces visual complaints, and may not be achievable in some patients, this may represent an important advantage of SD-OCT.

\section{Summary}

What was known before

- Some studies have indicated that time-domain OCT requires pupil dilation for the acquisition of good quality images of the retina and RNFL.

What this study adds

- The RNFL measurements obtained with spectral domain OCT are not influenced by pupil size.

\section{Conflict of interest}

The authors declare no conflict of interest.

\section{Acknowledgements}

This study was supported by Fundação de Amparo a Pesquisa do Estado de São Paulo (FAPESP), Grant no. 07/51281-9.

\section{References}

1 Quigley HA, Dunkelberger GR, Green WR. Chronic human glaucoma causing selectively greater loss of larger optic nerve fibers. Ophthalmology 1988; 95: 357-363.

2 Sommer A, Katz J, Quigley HA, Miller NR, Robin AL, Richter RC et al. Clinically detectable nerve fiber atrophy precedes the onset of glaucomatous field loss. Arch Ophthalmol 1991; 109: 77-83.

3 Read RM, Spaeth GL. The practical clinical appraisal of the optic disc in glaucoma: the natural history of cup progression and some disc-field correlations. Trans Am Acad Ophthalmol Otolaryngol 1974; 78: OP255-OP274.

4 Odberg T, Riise D. Early diagnosis of glaucoma: the value of successive stereophotography of the optic disc. Arch Ophthalmol (Copenh) 1985; 63: 257-263. 
5 Huang D, Swanson EA, Lin CP, Schuman JS, Stinson WG, Chang W et al. Optical coherence tomography. Science 1991; 254: 1178-1181.

6 Hee MR, Izatt JA, Swanson EA, Huang D, Schuman JS, Lin CP et al. Optical coherence tomography of the human retina. Arch Ophthalmol 1995; 113: 325-332.

7 Puliafito CA, Hee MR, Lin CP, Reichel E, Schuman JS, Duker JS et al. Imaging of macular diseases with optical coherence tomography. Ophthalmology 1995; 102: 217-229.

8 Drexler W, Morgner U, Ghanta RK, Kärtner FX, Schuman JS, Fujimoto JG. Ultra-high resolution ophthalmic optical coherence tomography. Nat Med 2001; 7: 502-507.

9 Lin SC, Singh K, Jampel HD, Hodapp EA, Smith SD, Francis BA et al. Optic nerve head and retinal nerve fiber layer analysis. Ophthalmology 2007; 114: 1937-1949.

10 Sanchez-Galeana C, Bowd C, Blumenthal EZ, Gokhale PA, Zangwill LM, Weinreb RN. Using imaging summary data to detect glaucoma. Ophthalmology 2001; 108: 1812-1818.

11 Smith M, Frost A, Graham CM, Shaw S. Effect of pupillary dilatation on glaucoma assessments using optical coherence tomography. $\mathrm{Br}$ J Ophthalmol 2007; 91: 1686-1690.

12 Paunescu LA, Schuman JS, Price LL, Stark PC, Beaton S, Ishikawa $\mathrm{H}$ et al. Reproducibility of nerve fiber thickness, macular thickness, and optic nerve head measurements using Stratus OCT. Invest Ophthalmol Vis Sci 2004; 45: 1716-1724.

13 Savini G, Zanini M, Barboni P. Influence of pupil size and cataract on retinal nerve fiber layer thickness measurements by stratus OCT. J Glaucoma 2006; 15: 336-340.

14 Ko TH, Fujimoto JG, Duker JS, Paunescu LA, Drexler W, Baumal CR et al. Comparison of ultra-high and standardresolution optical coherence tomography for imaging macular hole pathology and repair. Ophthalmology 2004; 111: 2033-2043.
15 Ko TH, Fujimoto JG, Schuman JS, Paunescu LA, Kowalevicz AM, Hartl I et al. Comparison of ultra-high and standard resolution optical coherence tomography for imaging of macular pathology. Ophthalmology 2005; 112: 1922.e1-1922.e15.

16 Gupta V, Gupta P, Singh R, Dogra MR, Gupta A. Spectraldomain Cirrus high-definition optical coherence tomography is better than time-domain Stratus optical coherence tomography for evaluation of macular pathologic features in uveitis. Am J Ophthalmol 2008; 145: 1018-1022.

17 Kiernan DF, Hariprasad SM, Chin EK, Kiernan CL, Rago J, Mieler WF. Prospective comparison of cirrus and stratus optical coherence tomography for quantifying retinal thickness. Am J Ophthalmol 2009; 147: 267-275.

18 Ho J, Sull AC, Vuong LN, Chen Y, Liu J, Fujimoto JG et al. Assessment of artifacts and reproducibility across spectraland time-domain optical coherence tomography devices. Ophthalmology 2009; 116: 1960-1970.

19 Schuman JS, Pedut-Kloizman T, Hertzmark E, Hee MR, Wilkins JR, Coker JG et al. Reproducibility of nerve fiber layer thickness measurements using optical coherence tomography. Ophthalmology 1996; 103: 1889-1898.

20 Schuman JS. Spectral domain optical coherence tomography for glaucoma (an AOS thesis). Trans Am Ophthalmol Soc 2008; 106: 426-458.

21 Zafar S, Gurses-Ozden R, Vessani R, Makornwattana M, Liebmann JM, Tello $C$ et al. Effect of pupillary dilation on retinal nerve fiber layer thickness measurements using optical coherence tomography. J Glaucoma 2004; 13(1): 34-37.

22 Hsu SY, Tsai RK. Analysis of retinal nerve fiber layer and macular thickness measurements in healthy Taiwanese individuals using optical coherence tomography (Stratus OCT). J Glaucoma 2008; 17(1): 30-35. 